\title{
用离子注人方法在 $\mathrm{SiO}_{2}$ 中制备纳米 $\alpha-\mathrm{Fe}$ 微晶及其性能研究 *
}

张桂林 刘文宏 徐 峰 胡文祥

(中国科学院上海原子核研究所, 上海 201800)

\section{关键词离子注人、纳米微晶 $\alpha-\mathrm{Fe}$ 、矫顽力}

纳米微晶材料的制备和它的性能研究, 是近几年来迅速发展起来的一门新学科. 现有气 凝、光化学和非晶晶化等多种制备纳米微晶方法. 文献 [1]首次报道了. 用 ${ }^{57} \mathrm{Fe}$ 离子注人 $\mathrm{Cu}$ 中 获得了纳米 $\alpha-\mathrm{Fe}$ 微晶. 该方法的原理是用强制的方法(离子注人), 使两种不固溶的元素镶 嵌在一起, 然后经高温退火, 使其中的注人元素偏析出来. 由于注人离子的深度只有几十纳 米, 所以偏析的微晶颗粒大小也只能在此范围之内. 本文报道了 $\mathrm{Fe}$ 离子注入 $\mathrm{SiO}_{2}$ 的结果, 借以制备很有应用前景的磁记录介质和光电器件复合材料. 我们用同位素分离器, 从天然铁 中分离出 ${ }^{57} \mathrm{Fe}$ 离子, 加速到 $60 \mathrm{keV}$ 的能量后, 注人 $\mathrm{CT}$ 切割的 $\mathrm{SiO}_{2}$ 单晶中, 注人剂量为 $5 \times$ $10^{16}$ 原子 $/ \mathrm{cm}^{2}$. 注入的样品在含微量氢的气氛中退火. 退火时所发生的动力学过程用内转换 电子 Mössbauer 谱 (CEMS) 和 Rutherford 背散射 (RBS) 进行测量, 所形成的 $\boldsymbol{\alpha}-\mathrm{Fe}$ 微晶 颗粒尺寸由透射电子显微镜 (TEM) 测得, 它的矫顽力 $H_{\mathrm{c}}$ 用振动样品磁强计测量.

\section{一、实验结果和讨论}

图 1 表示了刚注人和经不同温度退火后测得的 CEMS 谱. Mössbauer 源 为 ${ }^{57} \mathrm{Co}$ (Pd). 由图知, 在刚注人的样品中未出现铁磁相的六线谱。经最小二乘法拟合后, 该谱分解成 3 个亚 谱, 其中 1 个是单线谱 (S), 2 个为带有电四极矩分裂的双线谱 $\left(D_{1}\right.$ 和 $\left.D_{2}\right)$. 它们相对于标准 $\alpha-\mathrm{Fe}$ 的化学移分别为 $0.0 \mathrm{~mm} / \mathrm{s}, 0.38 \mathrm{~mm} / \mathrm{s}$ 和 $0.23 \mathrm{~mm} / \mathrm{s}$; 双线谱的电四极矩分裂值 分别为 $0.88 \mathrm{~mm} / \mathrm{s}$ 和 $0.76 \mathrm{~mm} / \mathrm{s}$. 这些参数经高温退火后略有变化, 但其相对强度显著减弱. 根据 Mössbauer 参数化学移值, 谱中的单线成分可认为来自夹杂在 $\mathrm{SiO}_{2}$ 晶格中具有超顺磁特性的 $\mathrm{Fe}$ 原子团簇 (cluster). 由于 $\mathrm{Fe}^{+}$离子的轰击, 部分 $\mathrm{O}-\mathrm{Si}-\mathrm{O}$ 链中的 $\mathrm{Si}-\mathrm{O}$ 键破裂, 而同 $\mathrm{Fe}$ 离子相连接形成 $\mathrm{Fe}-\mathrm{Si}-\mathrm{O}$ 型的复合体. 谱中 $\mathrm{D}_{1}$ 和 $\mathrm{D}_{2}$ 双线谱可能来自处于这种复合体 中具有不同价态的 $\mathrm{Fe}$ 离子 $\left(\mathrm{Fe}^{++}\right.$和 $\left.\mathrm{Fe}^{+++}\right)$的贡献 ${ }^{[2]}$.

当退火温度上升到 $650^{\circ} \mathrm{C}$, 保温 $30 \mathrm{~min}$ 后, 谱中明显出现具有大超晶细内场的六线谱型, 且可进一步分解成两组磁超精细内场, 其中一组的化学移和磁超精细内场值分别为 $0.07 \mathrm{~mm} / \mathrm{s}$ 和 $329 \times 10^{6} / 4 \pi \mathrm{A} / \mathrm{m}$ (即 $329 \mathrm{kOe}$ ). 这些参数, 以及它的线宽完全相似于多晶的 $\alpha-\mathrm{Fe}$ 值 (图 1(e)). 这表明该谱成分对应于具有体心立方结构的 $\alpha-\mathrm{Fe}$ 相, 其相对强度随退火时间的增加

1992-02-01 收稿, 1992-05-23 收修改稿

* 国家自然科学基金资助项目 
而增加, 且达到一饱和值. 另一组的化学移和磁超精细内场分 别为 $0.02 \mathrm{~mm} / \mathrm{s}$ 和 $288 \times 10^{6} /$ $4 \pi \mathrm{A} / \mathrm{m}$, 它的线宽明显地宽于上一组, 这表明它有一较宽的内场分布。浪据这些参数, 我们可

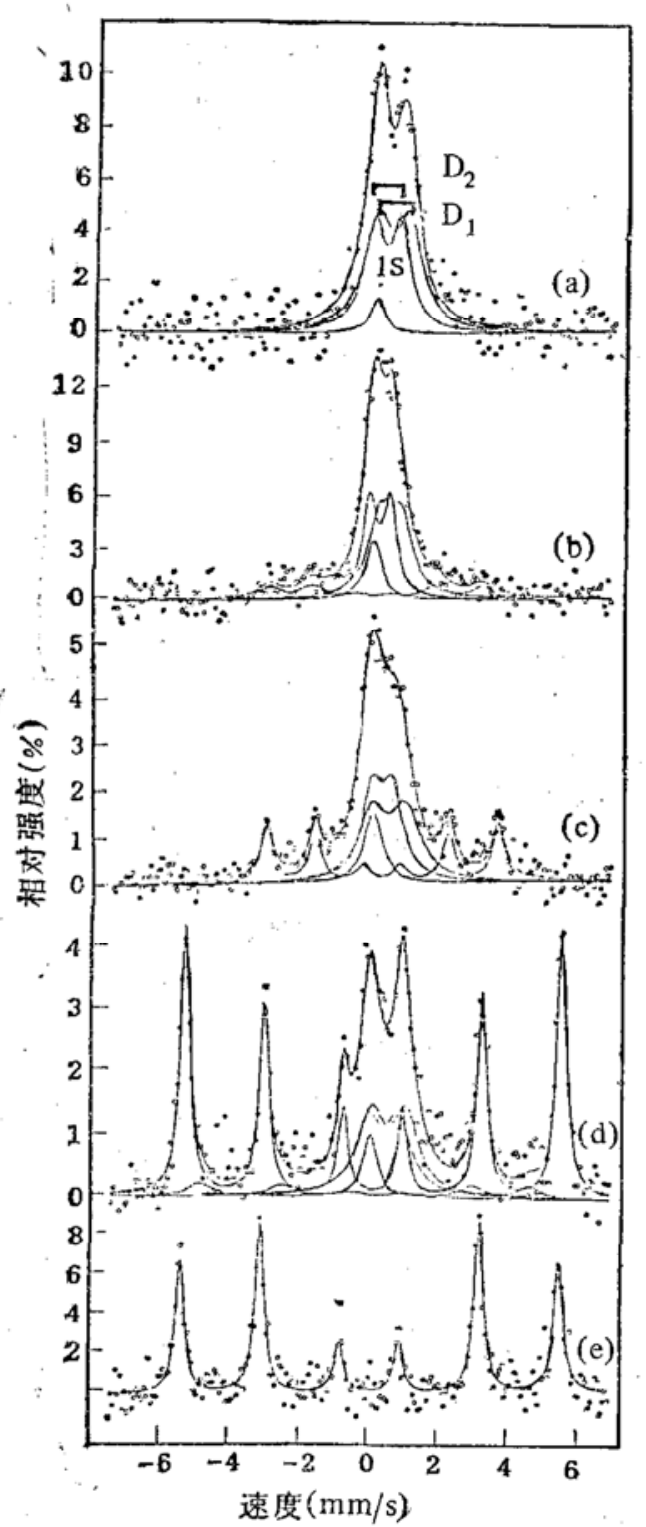

图 $1{ }^{57} \mathrm{Fe}$ 离子注入 $\mathrm{SiO}_{2}$ 经不同温度退 火后的 CEMS 谱

(a) 退火前, (b) $500^{\circ} \mathrm{C}, 30 \mathrm{~min}$, (c) $600^{\circ} \mathrm{C}$, $30 \mathrm{~min}$, (d) $650^{\circ} \mathrm{C}, 90 \mathrm{~min},(\mathrm{e}) \alpha-\mathrm{Fe}$. 注人能 珐: $60 \mathrm{keV}$ 以认为这组成分可能来自微晶 $\alpha-\mathrm{Fe}$ 的表面层 原子, 而这些微晶表面的 $\mathrm{Fe}$ 原子与 $\mathrm{Si}$ 原子相 邻. 已经知道, 每个最近邻 $\mathrm{Si}$ 原子将使 $\mathrm{Fe}$ 原 子的磁超精细场减少 $27 \times 10^{6} / 4 \pi \mathrm{A} / \mathrm{m}^{[3]}$. 一个 描述纳米微晶材料中界面原子的流行模 型等称 界面原子之间的距离应是随机分布的. 我们 获得的较宽的内场分布结果支持了这个模 型. 由此可见，具有磁超精细内场为 $329 \times 10^{6} / 4 \pi$. $\mathrm{A} / \mathrm{m}$ 的成分代表纳米 $\alpha-\mathrm{Fe}$ 颗粒的内部原子, 而平均内场为 $288 \times 10^{6} / 4 \pi \mathrm{A} / \mathrm{m}$ 的原子代表 与 $\mathrm{SiO}_{2}$ 祄底相接触的外层原子.

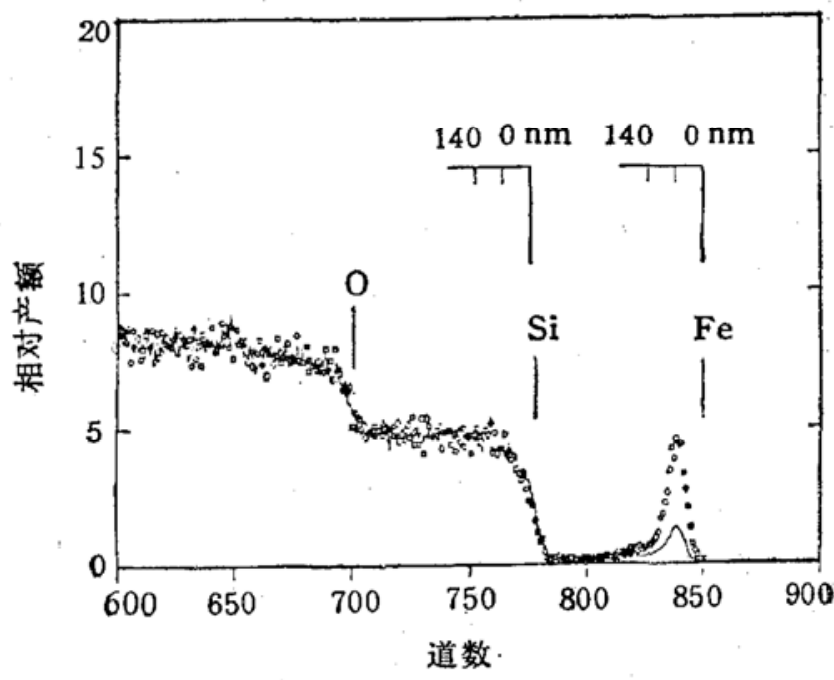

图 $2 \mathrm{Fe}$ 注入 $\mathrm{SiO}_{2}$ 样品在退火前后 的 RBS 谱

注人离子能星为 $60 \mathrm{keV}$. 唱为 $1 \times 10^{17} \mathrm{Fe} / \mathrm{cm}^{2}$, $700^{\circ} \mathrm{C}, 30 \mathrm{~min}$; 一一为 $1 \times 10^{16} \mathrm{Fe} / \mathrm{cm}^{2}$, 未退火

在出现 $329 \times 10^{6} / 4 \pi \mathrm{A} / \mathrm{m}$ 内场的成分前, 谱中出现小内场成分, 以及随后在稍高退火温 度 $\left(600^{\circ} \mathrm{C}\right)$ 下出现 $203 \times 10^{6} / 4 \pi \mathrm{A} / \mathrm{m}$ 的内场成分. 我们推测它们可能来自 $\alpha-\mathrm{Fe}$ 微晶的晶 化中心 ${ }^{[1]}$. 随退火温度的升高, $\alpha-\mathrm{Fe}$ 的结晶逐步趋于完整, 其中夹杂的 $\mathrm{Si}$ 原子逐步离析, 从 而内场逐渐增大。

图 2 表示了刚注入离子的样品和经高温退火形成了 $\alpha-\mathrm{Fe}$ 微晶的样品测得的 RBS 谱. 由 图 2 推知, 注人 $\mathrm{Fe}$ 离子的平均深度约为 $67 \mathrm{~nm}$, 深度分布的半宽度约为 $48 \mathrm{~nm}$; 经 $700^{\circ} \mathrm{C}$ 退火 $30 \mathrm{~min}$ 后, 其深度分布未发生变化. 这表明在此退火温度下 $\mathrm{Fe}$ 源子仅在注人层的范围内移 


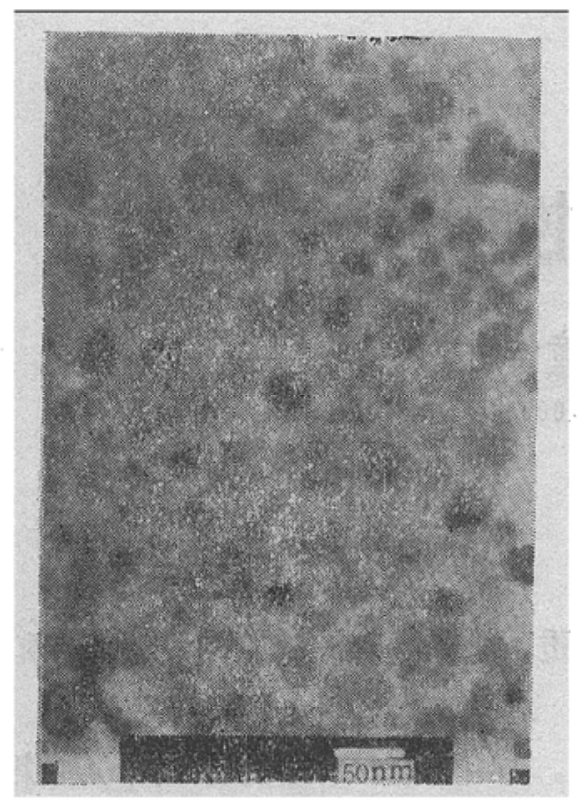

图 3 注入 $\mathrm{Fe}$ 在 $\mathrm{SiO}_{2}$ 中析出 $\alpha-\mathrm{Fe}$ 小颗粒的 TEM 图

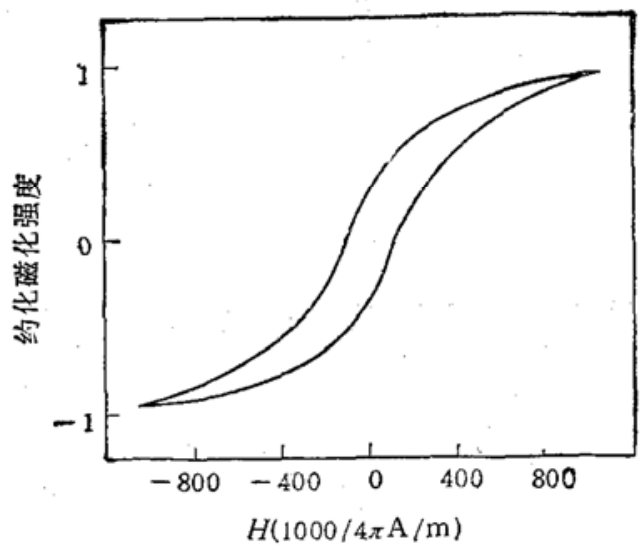

图 4 经退火后 $\mathrm{Fe}$ 注人 $\mathrm{SiO}_{2}$ 样品 的磁滞回线

动. $\mathrm{Fe}$ 原子之间的相互作用所产生的凝聚阻止了 $\mathrm{Fe}$ 原子的外扩散, 从而导致了 $\mathrm{Fe}$ 原子的偏 析和结晶. 由 TEM 测量的结果表明,所形成的 $\alpha-\mathrm{Fe}$ 影粒的平均尺寸大约为 $25 \mathrm{~nm}$ (图 3). 图 4 表示了由振动样品磁强计所测得的样品磁滞回线. 测得室温和 $78 \mathrm{~K}$ 时在 $\mathrm{SiO}_{2}$ 中 $\alpha-\mathrm{Fe}$ 颗粒的矫项力分别为 $100 \times 10^{3} / 4 \pi \mathrm{A} / \mathrm{m}$ 和 $70 \times 10^{3} / 4 \pi \mathrm{A} / \mathrm{m}$, 即室温时的 $H_{c}$ 比通常多晶 的 $\alpha-\mathrm{Fe}$ 的值 $\left(<0.8 \times 10^{3} 4 \pi \mathrm{A} / \mathrm{m}\right)$ 要大两个数量级. 奇怪的是, 在 $78 \mathrm{~K}$ 温度时, 测得的 $\boldsymbol{H}_{\mathrm{c}}$ 值 要比室温时略小. 按照 Kneller 和 Luborsky 公式 ${ }^{[5,6]}$, 对孤立的 $a-\mathrm{Fe}$ 小颗粓的矫顽力, 应 服从 $H_{\mathrm{c}}=H_{\mathrm{co}}\left(1-\sqrt{T / T_{\mathrm{g}}}\right), T_{\mathrm{g}}$ 为超顺磁的起始温度, 它正比于磁各向异性能 $\mathrm{K}$ 和颗粒的 体积 $\mathrm{V}$. 当温度升高时, 由于热振动的影响, 使单畴的磁矩变得不稳定, 从而使 $H_{\mathrm{c}}$ 下降. 我们 的结果却与此相反, 这值得进一步研究. 目前我们正在进行测量各向异性随温度的变化, 以期 得到这一现象的合理解释.

\section{参考文献}

[1] Zhang Guilin et a1., Phys. Lett, 119A(1986), 251-255.

[2] Bhagwat, S. et al., Phys. Rev., B40(1989), 700-703.

[3] Stearns, M. B., Phys, Rev., 147(1966), 439-453.

[ 4] Zhu, X. et al., Phys. Rev., B35(1987), 9085-9090.

[5] Kneller, E. F., Luborsky, F. E., J. Appi. Phys, 34(1963), 656-658.

[6] Xiao Gang, Chien, C. L., Appl. Phys. Lett, 51(1987),1280-1282. 